HD-THEP-01-11

hep-ph/0103049

March 2001

\title{
Nonequilibrium time evolution of the spectral function in quantum field theory
}

\author{
Gert Aarts* and Jürgen Berges ${ }^{\ddagger}$ \\ Institut für Theoretische Physik \\ Philosophenweg 16, 69120 Heidelberg, Germany
}

\begin{abstract}
Transport or kinetic equations are often derived assuming a quasiparticle (on-shell) representation of the spectral function. We investigate this assumption using a three-loop approximation of the $2 P I$ effective action in real time, without a gradient expansion or on-shell approximation. For a scalar field in $1+1$ dimensions the nonlinear evolution, including the integration over memory kernels, can be solved numerically. We find that a spectral function approximately described by a nonzero width emerges dynamically. During the nonequilibrium time evolution the Wigner transformed spectral function is slowly varying, even in presence of strong qualitative changes in the effective particle distribution. These results may be used to make further analytical progress towards a quantum Boltzmann equation including off-shell effects and a nonzero width.
\end{abstract}

PACS numbers: 11.10.Wx, 05.60.Gg, 05.70.Ln

*email: aarts@thphys.uni-heidelberg.de

‡email: j.berges@thphys.uni-heidelberg.de 


\section{Introduction}

Current and upcoming heavy-ion experiments at RHIC and LHC have been an important motivation for an extensive theoretical study of kinetic or transport equations beyond the level of the classical Boltzmann equation. In a (nonequilibrium) environment the quasiparticle structure of the theory can change and for instance the presence of nuclear matter in heavy-ion collisions may affect the mass and width of vector mesons (see e.g. [1] for a discussion of the broadening of the $\rho$ meson). In order to give a consistent treatment these effects have to be incorporated in a kinetic description. Also in other areas of physics an extension of the classical Boltzmann equation to a more general quantum Boltzmann equation is needed. The theory of baryo- and leptogenesis requires the solution of a large set of Boltzmann equations with a proper inclusion of CP violating and off-shell effects in an expanding universe [2]. In Ref. [3] a description of the dynamics of Bose-Einstein condensates using a quantum Boltzmann equation can be found.

It is well known [4, 5, 6, 7] that a consistent setup of kinetic equations derived from a microscopic quantum field theory needs to incorporate two quite different aspects: the statistical information typically described in terms of one-particle distribution functions on the one hand, and the kinetic information about the spectrum of the theory, encoded in the spectral function, on the other hand. There is a possibility to independently approximate the statistical and the spectral aspects of the time evolution equations. In the derivation of the classical Boltzmann equation one neglects all nontrivial kinetic aspects of the spectral function, which is approximated by an on-shell $\delta$-function. A first-order gradient expansion is then sufficient to derive the Boltzmann equation, describing the evolution of the statistical information only, from the underlying field theory $4,5,6,6,7$. It turns out that relaxing the assumption of on-shell quasi-particles makes the problem considerably more complicated. Indeed, an analysis of a self-consistent inclusion of a nonzero width has been taken up only relatively recently [8]. Similarly, to go beyond leading order in a gradient expansion seems to be rather cumbersome [9].

Recently, a successful numerical solution of the nonequilibrium time evolution without the use of a gradient expansion or on-shell approximation has been presented for a scalar field theory in $1+1$ dimensions [10]. Both the early-time behavior and the large-time physics of thermalization can be described remarkably well for quantum fields [10], as well as for the classical 
field limit put to the test by Monte Carlo methods and numerical integration [11]. The evolution equations can be obtained from the three-loop expansion of the closed time path generating functional for two-particle irreducible (2PI) Green's functions [5, 10] and comprise the classical Boltzmann equation [4, 月, 6, 7]. The full solution of these equations allows to directly investigate the influence of off-shell effects on the time evolution or the validity of a gradient expansion.

In this paper we focus on the kinetic aspects of the evolution equations and compute the nonequilibrium time dependence of the spectral function without further approximation. The paper is organized as follows. In the following section we briefly review the Schwinger-Keldysh formalism for a nonequilibrium scalar field. We derive exact time evolution equations starting from the Schwinger-Dyson equation for the two-point function and use the three-loop 2PI effective action to find a consistent approximation to the self energy. In Sec. 3 we discuss the initial conditions used in this paper. In order to make contact with heavy-ion physics we consider as initial state a so-called "tsunami": a particle number distribution peaked in momentum space. Such an initial state is reminiscent of two colliding high energy wave packets [12]. We hasten to say that in our $(1+1)$-dimensional scalar field model direct applicability to phenomology is of course limited. We study in this paper in particular the time evolution of the Wigner transformed spectral function suitable for comparison with standard frameworks for kinetic equations. Details of the Wigner transform are given in Sec. 1 . We present our numerical results in Sec. 5. In Sec. 6 we make a connection with equaltime methods and moment expansions employed before. Our conclusions are summarized in Sec. 0 .

\section{Time evolution equations}

Spectral function. We consider a real, scalar quantum field theory with field operators obeying the Heisenberg equation of motion $\left(\square_{x}+m^{2}\right) \phi(x)+$ $\lambda \phi^{3}(x) / 6=0$. We are interested in the nonequilibrium time evolution of the spectral function $\rho$ given by the expectation value of the commutator of two fields

$$
\rho(x, y)=i\left\langle[\phi(x), \phi(y)]_{-}\right\rangle .
$$

where $\langle\cdot\rangle$ denotes the average over an initial density matrix. Nonequilibrium 


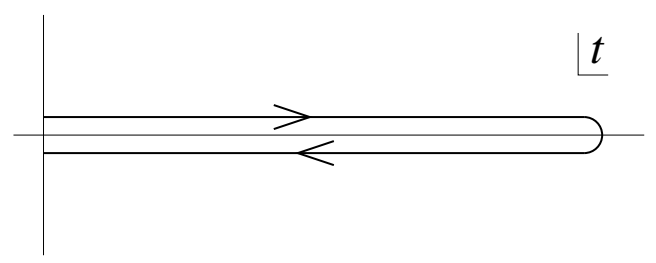

Figure 1: Keldysh contour $\mathcal{C}$ in the complex-time plane.

initial value problems can be formulated using the Schwinger-Keldysh or closed-time-path formalism [13]. For a detailed discussion we refer to Ref. [5]. In this formalism the theory is formulated along a contour $\mathcal{C}$ in the complextime plane, as shown in Fig. 1. Expectation values are defined along this contour. The two-point function is usually written as

$$
G(x, y)=G_{>}(x, y) \Theta_{\mathcal{C}}\left(x^{0}-y^{0}\right)+G_{<}(x, y) \Theta_{\mathcal{C}}\left(y^{0}-x^{0}\right)
$$

with $\Theta$-functions defined along the closed time path $\mathcal{C}$ and the Wightman functions defined as $G_{>}(x, y)=\langle\phi(x) \phi(y)\rangle=G_{<}(y, x)$. I For a real scalar field $G_{>}^{*}(x, y)=G_{<}(x, y)$. In terms of these, the spectral function is given by

$$
\rho(x, y)=i\left[G_{>}(x, y)-G_{<}(x, y)\right] .
$$

The spectral function can be obtained from the propagator (2.2) directly by writing

$$
G(x, y)=F(x, y)-\frac{i}{2} \rho(x, y)\left[\Theta_{\mathcal{C}}\left(x^{0}-y^{0}\right)-\Theta_{\mathcal{C}}\left(y^{0}-x^{0}\right)\right]
$$

Here we have separated the propagator using the commutator of two fields, $\rho(x, y)$, and the anti-commutator of two fields, the symmetric or statistical two-point function

$$
F(x, y)=\frac{1}{2}\left\langle[\phi(x), \phi(y)]_{+}\right\rangle=\frac{1}{2}\left[G_{>}(x, y)+G_{<}(x, y)\right] .
$$

For a real scalar field $F(x, y)$ and $\rho(x, y)$ are real functions with symmetry properties $F(x, y)=F(y, x)$ and $\rho(x, y)=-\rho(y, x)$. We use $\rho$ and $F$ as the basic quantities in this paper.

\footnotetext{
${ }^{1}$ These two-point functions were also used in Ref. 10.
} 
Evolution equations. The full propagator (2.4) obeys the SchwingerDyson equation

$$
G^{-1}(x, y)=G_{0}^{-1}(x, y)-\Sigma(x, y)
$$

with the proper self energy $\Sigma$ and $G_{0}^{-1}(x, y)=i\left[\square_{x}+m^{2}\right] \delta_{\mathcal{C}}^{d+1}(x-y)$. A differential equation for $G$ which may be used to study the time evolution for given initial conditions can be obtained from (2.6) using $\int_{\mathcal{C}} d^{d+1} z G^{-1}(x, z) G(z, y)=$ $\delta_{\mathcal{C}}^{d+1}(x-y)$ and one finds

$$
\left[\square_{x}+M^{2}(x)\right] G(x, y)+i \int_{\mathcal{C}} d^{d+1} z \Sigma^{(\text {nonlocal })}(x, z) G(z, y)=-i \delta_{\mathcal{C}}^{d+1}(x-y) .
$$

Here we have separated $\Sigma$ in a local part and a nonlocal part,

$$
\Sigma(x, y)=-i \Sigma^{(\text {local })}(x) \delta_{\mathcal{C}}^{d+1}(x-y)+\Sigma^{(\text {nonlocal })}(x, y) .
$$

The local part $\Sigma^{\text {(local) }}$ corresponds to a mass shift and we find it convenient to write $M^{2}(x)=m^{2}+\Sigma^{(\text {local })}(x)$.

Since our interest focuses on the nonequilibrium dynamics of $\rho$ we rewrite (2.7) such that the spectral function appears explicitly as one of the dynamical degrees of freedom. Similar to the above decomposition for $G$ we can decompose the self energy

$$
\Sigma^{(\text {nonlocal })}(x, y)=\Sigma_{F}(x, y)-\frac{i}{2} \Sigma_{\rho}(x, y)\left[\Theta_{\mathcal{C}}\left(x^{0}-y^{0}\right)-\Theta_{\mathcal{C}}\left(y^{0}-x^{0}\right)\right]
$$

into two real functions, the statistical component of the self energy obeying $\Sigma_{F}(x, y)=\Sigma_{F}(y, x)$ and the spectral component $\Sigma_{\rho}(x, y)=-\Sigma_{\rho}(y, x)$. These self energies are related to the usual $\Sigma_{>,<}$as

$$
\begin{aligned}
\Sigma_{\rho}(x, y) & =i\left[\Sigma_{>}(x, y)-\Sigma_{<}(x, y)\right] \\
\Sigma_{F}(x, y) & =\frac{1}{2}\left[\Sigma_{>}(x, y)+\Sigma_{<}(x, y)\right] .
\end{aligned}
$$

We also note that the retarded and advanced propagators and self energies are related to the spectral components as $G_{R}(x, y)=\Theta\left(x^{0}-y^{0}\right) \rho(x, y)=$ $G_{A}(y, x)$ and $\Sigma_{R}(x, y)=\Theta\left(x^{0}-y^{0}\right) \Sigma_{\rho}(x, y)=\Sigma_{A}(y, x)$.

It is straightforward to express (2.7) in terms of the real functions introduced above. The resulting equations are

$$
\left[\square_{x}+M^{2}(x)\right] \rho(x, y)=-\int_{y^{0}}^{x^{0}} d z^{0} \int d \mathbf{z} \Sigma_{\rho}(x, z) \rho(z, y),
$$




$$
\begin{aligned}
{\left[\square_{x}+M^{2}(x)\right] F(x, y)=} & -\int_{0}^{x^{0}} d z^{0} \int d \mathbf{z} \Sigma_{\rho}(x, z) F(z, y) \\
& +\int_{0}^{y^{0}} d z^{0} \int d \mathbf{z} \Sigma_{F}(x, z) \rho(z, y) .
\end{aligned}
$$

The form of these equations is exact and they are equivalent to the standard Schwinger-Dyson identity (2.6). The equations for $\rho$ and $F$ are explicitly real and causal. A generic feature is the presence of nonlocal "memory" integrals. The time integral in Eq. (2.11) starts at some initial time taken to be $z^{0}=0$. We note that due to the canonical commutation relations the spectral function obeys the equal-time properties

$$
\left.\rho(x, y)\right|_{x^{0}=y^{0}}=0,\left.\quad \partial_{x^{0}} \rho(x, y)\right|_{x^{0}=y^{0}}=\delta^{d}(\mathbf{x}-\mathbf{y}) .
$$

The time evolution of the spectral function is completely determined by (2.10) and (2.12).

Three-loop 2PI effective action. To solve the Eqs. (2.10) and (2.11) one has to find suitable approximation schemes for the self energies $\Sigma_{F}$ and $\Sigma_{\rho}$ which respect all symmetries and allow to describe the nonequilibrium earlytime behavior as well as the large-time approach to equilibrium. Time reversal symmetry can be easily guaranteed by deriving the evolution equations from an effective action by a variational principle. A systematic way to achieve the latter is by using the loop expansion of the $2 P I$ effective action [14], formulated along the closed time path [15, 5]. Recently, it has been shown that the equations in the three-loop approximation describe both the early-time behavior and the physics of thermalization [10, 11]. The self energies in this approximation [5, 10], here expressed in terms of $\rho$ and $F$, are for a Gaussian initial density matrix given by

$$
\begin{aligned}
\Sigma_{\rho}(x, z) & =-\frac{\lambda^{2}}{2} \rho(x, z)\left[F^{2}(x, z)-\frac{1}{12} \rho^{2}(x, z)\right], \\
\Sigma_{F}(x, z) & =-\frac{\lambda^{2}}{6} F(x, z)\left[F^{2}(x, z)-\frac{3}{4} \rho^{2}(x, z)\right]
\end{aligned}
$$

(cf. Ref. [10] for the extension to quartic initial density matrices). The effective mass term including the local part of the self energy reads

$$
M^{2}(x)=m^{2}+\frac{\lambda}{2} F(x, x) .
$$


Eqs. (2.10, 2.11) with (2.13 2.15) form a closed set for $\rho$ and $F$ that is energy conserving and time-reversal invariant. We emphasize that the propagators in the "tadpole" diagram contribution in Eq. (2.15) and in the "settingsun" contributions (2.13, 2.14) are full ones. The form of the self energies presented here (with free instead of full propagators) has also been found in a perturbative calculation in thermal equilibrium [16]. We note from Eqs. (2.10, 2.11) that in local approximations, such as the Hartree approximation, derivable from the two-loop $2 P I$ effective action, and leading-order large $N$ schemes, the evolution of the spectral function $\rho$ does not couple back to the evolution of the symmetric two-point function $F$.

Thermal equilibrium. It is instructive to consider the introduced quantities in thermal equilibrium. The two-point function $G$ in equilibrium is described by the same equation (2.7) if the closed time path is replaced by an imaginary path $\mathcal{C}=[0,-i \beta]$, with $\beta$ the inverse temperature [4]. Since the equilibrium two-point functions depend only on the relative coordinates it is convenient to consider the Fourier transforms $\rho^{(\mathrm{eq})}(\omega, \mathbf{p})$, etc. From the periodicity ("KMS") condition for $G(x, y)$ and $\Sigma^{\text {(nonlocal) }}(x, y)$ in imaginary time one finds the generic equilibrium relations relating for example $G_{>}^{(\mathrm{eq})}(\omega, \mathbf{p})$ and $G_{<}^{(\mathrm{eq})}(\omega, \mathbf{p})$ [17:

$$
G_{>}^{(\mathrm{eq})}(\omega, \mathbf{p})=e^{\beta \omega} G_{<}^{(\mathrm{eq})}(\omega, \mathbf{p})
$$

For the spectral and statistical components we employ here this translates into the following equilibrium relations [16]

$$
\begin{aligned}
& F^{(\mathrm{eq})}(\omega, \mathbf{p})=-i\left(n_{\mathrm{B}}(\omega)+\frac{1}{2}\right) \rho^{(\mathrm{eq})}(\omega, \mathbf{p}), \\
& \Sigma_{F}^{(\mathrm{eq})}(\omega, \mathbf{p})=-i\left(n_{\mathrm{B}}(\omega)+\frac{1}{2}\right) \Sigma_{\rho}^{(\mathrm{eq})}(\omega, \mathbf{p})
\end{aligned}
$$

with $n_{\mathrm{B}}(\omega)=\left(e^{\beta \omega}-1\right)^{-1}$. While the spectral function $\rho^{(\mathrm{eq})}$ encodes the information about the spectrum, one observes that the symmetric function $F^{\text {(eq) }}$ encodes the statistical aspects in terms of the particle distribution function $n_{\mathrm{B}}$. For a vanishing $\omega$-dependence the function $\Gamma^{(\mathrm{eq})}(\omega, \mathbf{p}) \equiv \Sigma_{\rho}^{(\mathrm{eq})}(\omega, \mathbf{p}) / 2 \omega$ plays the role of a decay rate for one-particle excited states with momentum $\mathbf{p}$. 


\section{Initial conditions}

We consider a spatially homogeneous system such that, after spatial Fourier transformation, the two-point functions depend only on $\mathbf{p}$ and we consider the modes $\rho\left(x^{0}, y^{0} ; \mathbf{p}\right)$. As indicated above, the initial conditions for $\rho\left(x^{0}, y^{0} ; \mathbf{p}\right)$ are fixed by the equal-time relations (2.12). As initial conditions for the symmetric two-point function $F\left(x^{0}, y^{0} ; \mathbf{p}\right)$ we consider a "tsunami"-like situation [12] in which a spatially homogeneous collection of particles move with approximately the same momentum peaked around $\mathbf{p}_{\mathrm{ts}}$ and $-\mathbf{p}_{\mathrm{ts}}$. Explicitly, we take

$$
\begin{aligned}
\left.F_{0}\left(x^{0}, y^{0} ; \mathbf{p}\right)\right|_{x^{0}=y^{0}=0} & =\frac{1}{\omega_{\mathbf{p}}}\left[n_{0}(\mathbf{p})+\frac{1}{2}\right], \\
\left.\partial_{x^{0}} \partial_{y^{0}} F_{0}\left(x^{0}, y^{0} ; \mathbf{p}\right)\right|_{x^{0}=y^{0}=0} & =\omega_{\mathbf{p}}\left[n_{0}(\mathbf{p})+\frac{1}{2}\right], \\
\left.\partial_{x^{0}} F_{0}\left(x^{0}, y^{0} ; \mathbf{p}\right)\right|_{x^{0}=y^{0}=0} & =0,
\end{aligned}
$$

with $\omega_{\mathbf{p}}=\sqrt{\mathbf{p}^{2}+m^{2}}$ and the initial particle number

$$
n_{0}(\mathbf{p})=\mathcal{N} \exp \left(-\frac{1}{2 \sigma^{2}}\left(|\mathbf{p}|-\left|\mathbf{p}_{\mathrm{ts}}\right|\right)^{2}\right) .
$$

Here $\sigma$ controls the width of the initial distribution and $\mathcal{N}$ is a normalization constant. The initial condition is clearly far from thermal equilibrium and reminiscent of two colliding high energy wave packets [12].

To study the nonequilibrium evolution of the spectral function $\rho\left(x^{0}, y^{0} ; \mathbf{p}\right)$ we solve the coupled Eqs. (2.10 2.11) with (2.13 2.15) numerically along the lines of ref. [10]. We use a standard discretization on a lattice in space and time for a finite spatial volume and spatially periodic boundary conditions. To remove finite size effects we increase the volume until the results become stable. Because the evaluation of the memory integrals is rather time- and memory-consuming the numerical results presented below are for a $(1+1)$-dimensional system. The lattice acts as a cutoff and regulates the ultraviolet divergences, which have to be renormalized. In $1+1$ dimensions this is straightforward since only the one-loop contribution is logarithmically divergent. We absorb this in a bare mass parameter $\mu$ with the replacement $m^{2} \mapsto \mu^{2}=m^{2}-\delta m^{2}$ in Eq. (2.15). The counterterm $\delta m^{2}$ cancels the divergent vacuum contribution coming from the one-loop graph. The finite part 
of $\delta m^{2}$ is fixed by requiring that the renormalized one-loop mass parameter in vacuum $\left(n_{0}(\mathbf{p}) \equiv 0\right)$ equals $m$ and we express all dimensionful scales in units of $m$. For the plots we have used a space lattice with spacing $m a=0.3$ and a time lattice $a_{0} / a=0.25$. The system size is $m L=24$.

\section{Wigner transformation}

Derivations of transport or kinetic equations are typically performed by considering correlation functions in the so-called Wigner representation. Twopoint functions are written in terms of the center-of-mass coordinate $X=$ $(x+y) / 2$ and the relative coordinate $x-y$, with respect to which a Fourier transformation to momentum space is performed (Wigner transformation). Derivations based on a gradient expansion in $\partial_{X}$ assume a slow variation of the two-point functions with $X$. In particular, the classical Boltzmann equation can be obtained from (2.10 2.15) using a first-order gradient expansion and an on-shell form of the spectral function with zero width [5]. The full equations 2.10 2.15) can therefore be considered as a "quantum Boltzmann equation" including off-shell effects and resumming an infinite order of derivatives.

To analyze the evolution of the spectral function (not to solve the dynamics) we perform a Wigner transformation and write

$$
i \rho\left(X^{0} ; \omega, \mathbf{p}\right)=\int_{-2 X^{0}}^{2 X^{0}} d t e^{i \omega t} \rho\left(X^{0}+t / 2, X^{0}-t / 2 ; \mathbf{p}\right) .
$$

The $i$ is introduced such that $\rho\left(X^{0} ; \omega, \mathbf{p}\right)$ is real. Because the spectral function is antisymmetric, $\rho\left(X^{0} ; \omega, \mathbf{p}\right)=-\rho\left(X^{0} ;-\omega, \mathbf{p}\right)$, we will present the positive-frequency part only. Furthermore, as a consequence of the equal-time relation (2.12) the Wigner transform obeys the sum rule $I=$ $\int d \omega /(2 \pi) \omega \rho\left(X^{0} ; \omega, \mathbf{p}\right)=1$. In our simulations an evaluation of the sum rule typically results in $1.000<I<1.003$, indicating the accuracy in the numerical evaluation of the Wigner transform.

Since we consider an initial-value problem with $x^{0}, y^{0} \geq 0$, the time integral over $t=x^{0}-y^{0}$ is bounded by $\pm 2 X^{0}$. This leads to a so-called "finite-time effect" in the Wigner transformed quantities. To understand the qualitative aspects of a finite-time interval it is instructive to consider the free 
spectral function $\rho_{0}\left(x^{0}, y^{0} ; \mathbf{p}\right)=\omega_{\mathbf{p}}^{-1} \sin \left[\omega_{\mathbf{p}}\left(x^{0}-y^{0}\right)\right]$ with $\omega_{\mathbf{p}}=\sqrt{\mathbf{p}^{2}+m^{2}}$. After performing a Wigner transformation, we find

$$
\rho_{0}\left(X^{0} ; \omega, \mathbf{p}\right)=\frac{\sin \left[\left(\omega-\omega_{\mathbf{p}}\right) 2 X^{0}\right]}{\omega_{\mathbf{p}}\left(\omega-\omega_{\mathbf{p}}\right)}-\frac{\sin \left[\left(\omega+\omega_{\mathbf{p}}\right) 2 X^{0}\right]}{\omega_{\mathbf{p}}\left(\omega+\omega_{\mathbf{p}}\right)} .
$$

For finite $X^{0}$ this spectral function shows a rapidly oscillating behavior, while its envelope is peaked at $\omega= \pm \omega_{\mathbf{p}}$. In the limit $X^{0} \rightarrow \infty$ it reduces to $\rho_{0}(\omega, \mathbf{p})=2 \pi \operatorname{sgn}(\omega) \delta\left(\omega^{2}-\omega_{\mathbf{p}}^{2}\right)$.

For an interacting system, however, effective damping leads to a finite correlation time $\tau$, such that finite-time effects are expected to vanish when $X^{0} \gg \tau$. This can be investigated analytically by assuming that the spectral function is strictly exponentially damped and can be written as $\rho\left(x^{0}, y^{0} ; \mathbf{p}\right)=$ $e^{-\gamma_{\mathbf{p}}\left|x^{0}-y^{0}\right|} E_{\mathbf{p}}^{-1} \sin \left[E_{\mathbf{p}}\left(x^{0}-y^{0}\right)\right]$. The effective mass $E_{\mathbf{p}}$ and rate $\gamma_{\mathbf{p}}$ are allowed to depend on the time $X^{0}$. The corresponding Wigner transform reads $\rho\left(X^{0} ; \omega, \mathbf{p}\right)=\rho_{\mathrm{BW}}\left(X^{0} ; \omega, \mathbf{p}\right)+\delta \rho\left(X^{0} ; \omega, \mathbf{p}\right)$, where $\rho_{\mathrm{BW}}$ denotes the Breit-Wigner function

$$
\rho_{\mathrm{BW}}\left(X^{0} ; \omega, \mathbf{p}\right)=\frac{2 \omega \Gamma_{\mathbf{p}}\left(X^{0}\right)}{\left[\omega^{2}-E_{\mathbf{p}}^{2}\left(X^{0}\right)\right]^{2}+\omega^{2} \Gamma_{\mathbf{p}}^{2}\left(X^{0}\right)}
$$

with a width $\Gamma_{\mathbf{p}}\left(X^{0}\right)=2 \gamma_{\mathbf{p}}\left(X^{0}\right)$. The additional contribution $\delta \rho\left(X^{0} ; \omega, \mathbf{p}\right)$ vanishes exponentially as $\exp \left(-\Gamma_{\mathbf{p}} X^{0}\right)$, such that the finite-time effect is indeed absent for large enough (but finite) $X^{0}$. We want to emphasize that this finite-time effect is a general consequence of an initial-time problem formulated in terms of Wigner transformed quantities and should be taken into account when used to describe the early-time behaviour $\left(\Gamma_{\mathbf{p}} X^{0} \lesssim 1\right)$ in an experimental setup.

Finally, we note that the positivity condition, $\operatorname{sgn}(\omega) \rho\left(X^{0} ; \omega, \mathbf{p}\right) \geq 0$, can only be shown to hold in the special case that the initial density matrix commutes with the full Hamiltonian, such as in thermal equilibrium. In this case the system is of course stationary and independent of $X^{0}$. As a consequence the interpretation of the nonequilibrium spectral function as the density of states should be taken with care.

\section{Nonequilibrium evolution}

In Fig. 2 we show the time evolution of the spectral function $\rho\left(X^{0}+t / 2, X^{0}-\right.$ $t / 2 ; p)$ as a function of the relative time $t$ for two different momentum modes 


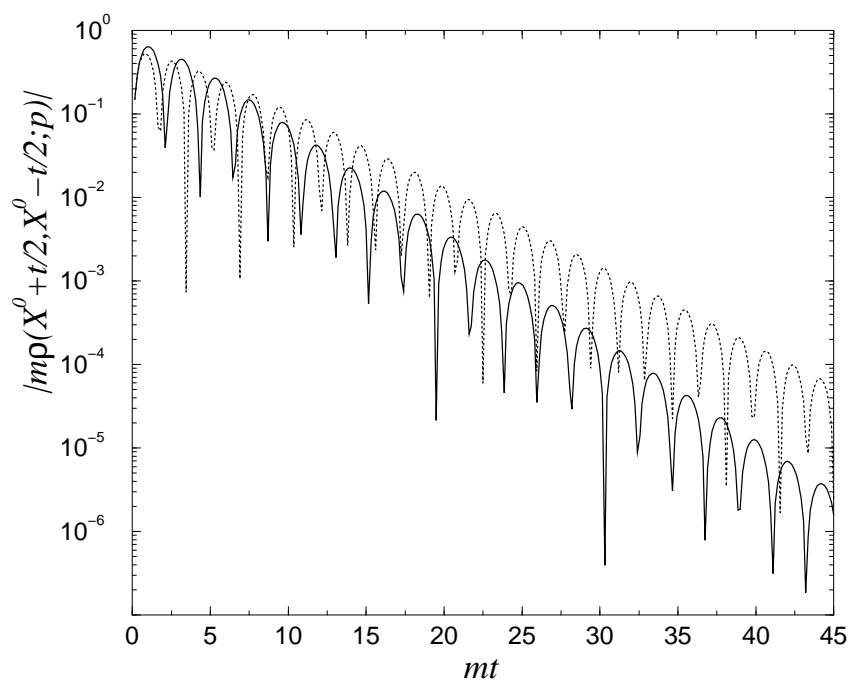

Figure 2: Absolute value of $\rho\left(X^{0} / 2+t, X^{0} / 2-t ; p\right)$ as a function of $t$ at fixed $m X^{0}=35.1$ for spatial momentum $p=2 \pi k / L$ with $k=0$ (full), 4 (dotted). The coupling is $\lambda / m^{2}=4$.

at given $X^{0}$, as obtained from the numerical solution of Eqs. (2.10 2.15). From the logarithmic plot one observes an effective damping of the oscillating spectral function while the oscillations never damp out completely. The decrease of the maximum amplitude very quickly approaches an approximately exponential behavior characteristic for the decay of one-particle excited states with given momentum. One observes that the zero momentum mode is stronger damped than the higher momentum mode.]

The effective damping should correspond to a nonzero width of the Wigner transformed spectral function. In Fig. 政 we display the Wigner transforms $\rho\left(X^{0} ; \omega, p\right)$ for the zero momentum mode (left peak) and the higher momentum mode (right peak) for the same $X^{0}$-time as in Fig. 2. One clearly observes that the interacting theory has a continuous spectrum described by a peaked spectral function with a nonzero width. The inset shows a blow-up of the zero mode around the three-particle threshold $3 E_{p} / m=4.38$. The expected bump in the spectral function is small but visible. We stress that

\footnotetext{
[10].

${ }^{2}$ An analysis of damping times as a function of the coupling $\lambda$ can be found in Ref.
} 


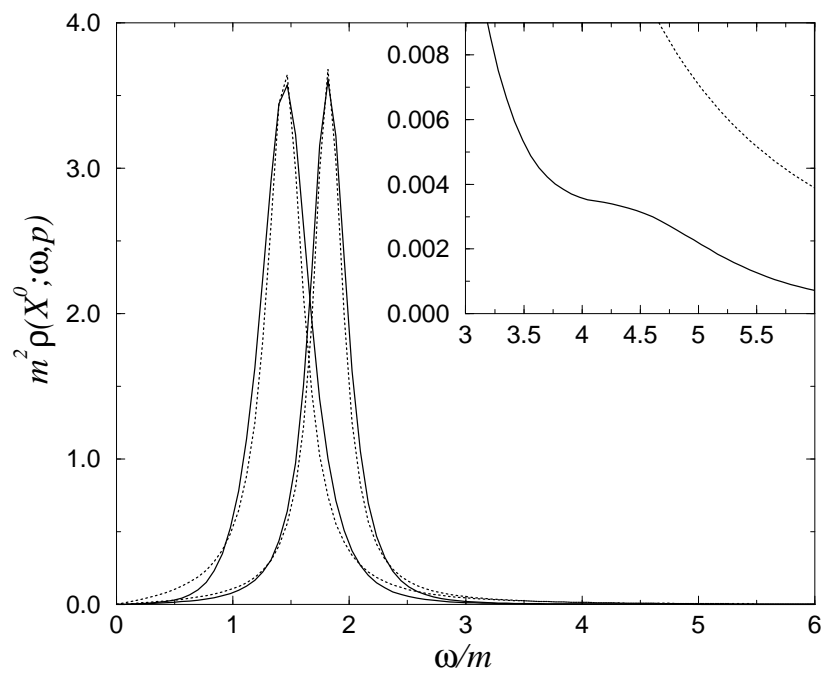

Figure 3: Wigner transforms $\rho\left(X^{0} ; \omega, p\right)$ of the real-time spectral functions of Fig. 2 as a function of $\omega$ at $m X^{0}=35.1$ for $k=0$ (left peak) and $k=4$ (right peak). Also shown are fits to a Breit-Wigner function (dotted) with $\left(E_{p} / m, \Gamma_{p} / m\right)=(1.46,0.37)(k=0)$ and $(1.82,0.30)(k=4)$. The inset shows a blow-up of the zero mode around the three-particle threshold $3 E_{p} / m=4.38$. The expected bump from off-shell decay is small but visible.

this bump in the spectral function is kinematically forbidden for the on-shell limit and arises purely from off-shell decay. In Fig. 3 we also present fits to a Breit-Wigner spectral function. While the position of the peak can be fitted easily, the overall shape and width are only qualitatively captured. In particular, the slope of $\rho\left(X^{0} ; \omega, p\right)$ for small $\omega$ is quantitatively different. We also see that the Breit-Wigner fits give a narrower spectral function (smaller width) and therefore would predict a slower exponential relaxation in real time.

We now turn to the dependence of $\rho\left(X^{0} ; \omega, p\right)$ on $X^{0}$. In Fig. 4 we show the spectral function at zero momentum $\rho\left(X^{0} ; \omega, p=0\right)$ for three different values of $X^{0}$. We see that the peak slowly shifts to smaller values. In the inset to Fig. 1 this behavior is quantified by plotting the $X^{0}$-dependence of $E_{p}\left(X^{0}\right)$ obtained from a fit to the Breit-Wigner function (4.3). For comparison, the solid line in the same figure shows the time-dependent one-particle energy 


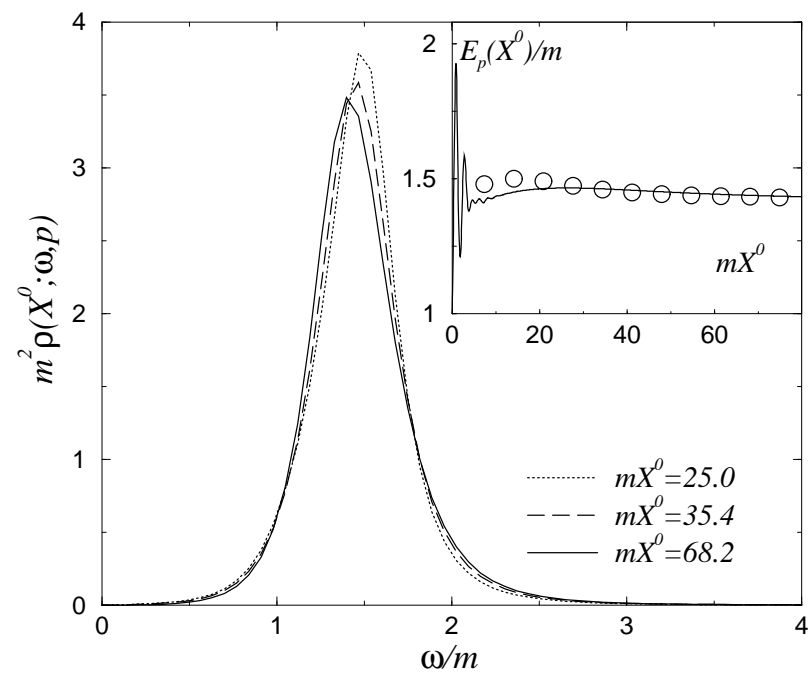

Figure 4: Time dependence of the spectral function $\rho\left(X^{0} ; \omega, p\right)$ at three values of $X^{0}$ for $p=0$. The peak slowly shifts towards lower values. The inset shows the $X^{0}$-dependence of $E_{p}\left(X^{0}\right)$ obtained from a fit to a Breit-Wigner function (circles) as well as the one-particle energy $\varepsilon_{p}\left(X^{0}\right)$ defined via equal-time twopoint functions, see Eq. (5.2) below (line).

$\epsilon_{p}\left(X^{0}\right)$ as defined via equal-time two-point functions in Eq. (5.2) below. We see a good agreement of both definitions of the one-particle energy for large enough times. We come back to this below.

A qualitative way to characterize the $X^{0}$-dependence of the shape of the spectral function is to assume that it is approximately described by a BreitWigner function. The time dependence of $\Gamma_{p}\left(X^{0}\right)$ can then be followed by performing fits for different values of $X^{0}$. The resulting $\Gamma_{p}\left(X^{0}\right) / 2$ is shown in Fig. 5 for two momentum modes. After a variation for early times, the $X^{0}$-dependence becomes weaker for larger times. As indicated before (see Fig. (3), the Breit-Wigner fits result in a smaller width than what is expected by comparing with the actual spectral function. A simple way to obtain a quantitative description is by extracting a relaxation rate $\gamma_{p}$ directly in real time, i.e. from a fit of the spectral function $\rho\left(X^{0}+t / 2, X^{0}-t / 2 ; p\right)$ to an exponential form $\sim \exp \left[-\gamma_{p}\left(X^{0}\right) t\right]$ (see Fig 2). The resulting rates are also presented in Fig. 5. We observe that the relaxation rate for the zero mode 


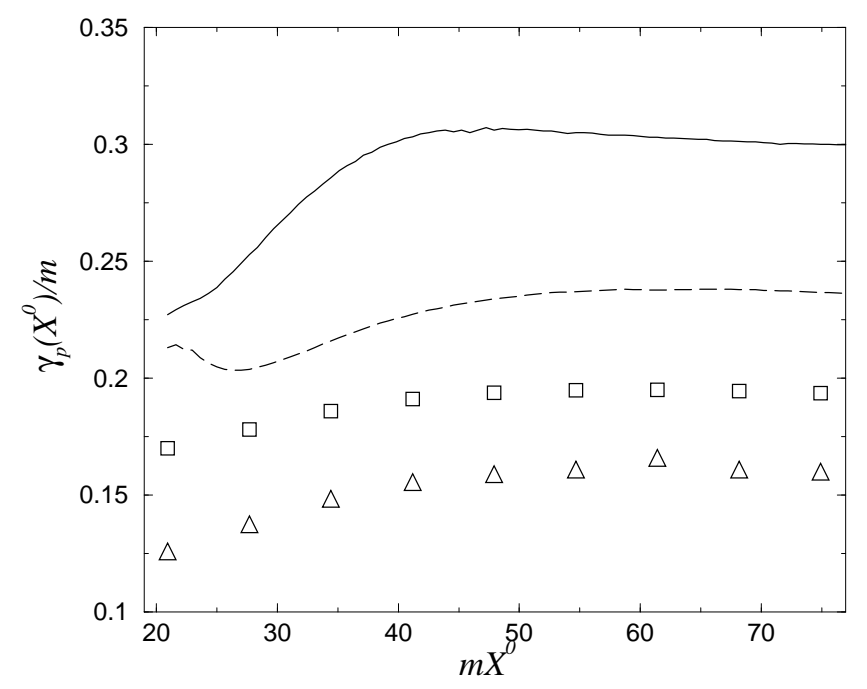

Figure 5: Time dependence of the relaxation rate $\gamma_{p}\left(X^{0}\right)$, determined directly in real time from $\rho\left(X^{0}+t / 2, X^{0}-t / 2 ; p\right)$ for $p=2 \pi k / L, k=0$ (full) and $k=4$ (dashed). Also shown is $\Gamma_{p}\left(X^{0}\right) / 2$ from a fit to a Breit-Wigner function, for $k=0$ (squares) and $k=4$ (triangles).

increases first, corresponding to a broadening of the Wigner transformed spectral function, before it levels off at later times. Despite the quantitative differences we see a similar qualitative $X^{0}$-dependence from the Breit-Wigner fit for large enough times.

Particle distribution function. The relatively weak $X^{0}$-dependence of the kinetic aspects of the evolution encoded in the spectral function has to be confronted with the time dependence of the statistical aspects encoded in $F$. The particle distribution is expected to exhibit strong qualitative changes, since we start with an initial condition (3.2) far away from thermal equilibrium and the current approximation is known to describe the physics of thermalization at large enough times [10].

The evolution of the statistical information can be extracted from the equal-time correlation function $F(t, t ; p)$. If the theory exhibits a quasiparticle structure it is possible to define a quasi-particle distribution function from a combination of equal-time two-point functions [18, 19]. For this purpose we introduce the two-point function $K(x, y)=\frac{1}{2}\left\langle[\pi(x), \pi(y)]_{+}\right\rangle$, where 


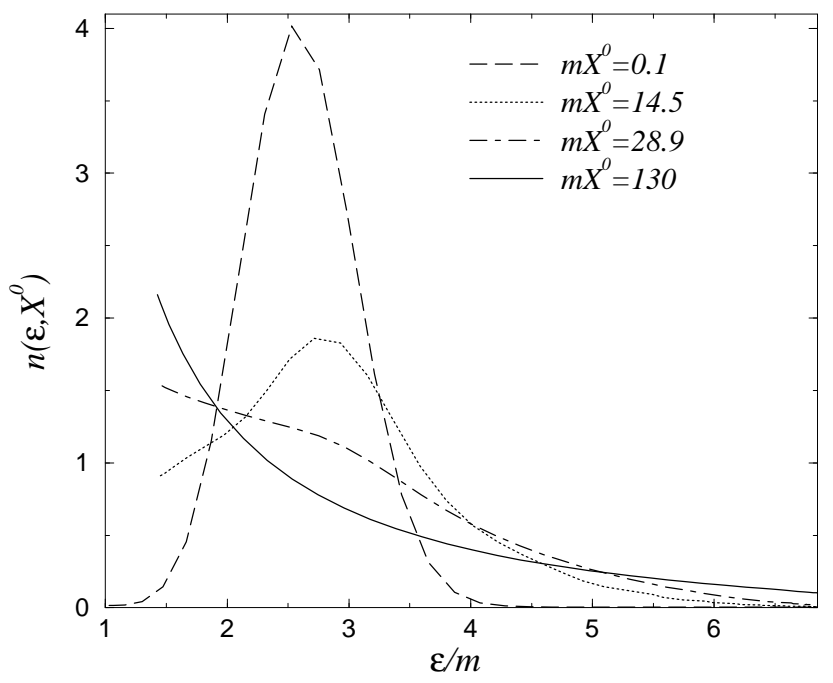

Figure 6: Effective particle number $n\left(\varepsilon_{p}, X^{0}\right)$ as a function of $\varepsilon_{p}$ at four values of $X^{0}$. As time goes on, the original "tsunami" vanishes and the infrared modes become more excited.

$\pi(x)=\partial_{x^{0}} \phi(x)$ is the canonical momentum. In the spatially homogeneous situation we consider here, $K\left(x^{0}, y^{0} ; p\right)=\partial_{x^{0}} \partial_{y^{0}} F\left(x^{0}, y^{0} ; p\right)$. We define the distribution function $n\left(\varepsilon_{p}\left(X^{0}\right), X^{0}\right)$ at time $X^{0}$ as

$$
n\left(\varepsilon_{p}, X^{0}\right)+\frac{1}{2}=\left[K\left(X^{0}, X^{0} ; p\right) F\left(X^{0}, X^{0} ; p\right)\right]^{1 / 2} .
$$

For the free theory the definition of $n\left(\varepsilon_{p}, X^{0}\right)$ corresponds to the standard one-particle distribution. The distribution function depends on a time-dependent one-particle energy, defined as

$$
\varepsilon_{p}\left(X^{0}\right)=\left[K\left(X^{0}, X^{0} ; p\right) / F\left(X^{0}, X^{0} ; p\right)\right]^{1 / 2} .
$$

The applicability of this definition to describe one-particle energies for the present nonequilibrium situation finds an impressive demonstration by comparing it with the position of the peak in the spectral function as discussed above (cf. Fig. 田).

In Fig. 60 we show the results for the effective particle number $n\left(\varepsilon_{p}, X^{0}\right)$ as a function of $\varepsilon_{p}$ at four values of $X^{0}$. The initially high occupation number in 
a small momentum range rearranges as time goes on. The original "tsunami" vanishes and the infrared modes become more excited. The distribution is not yet thermal. Thermalization takes place on longer time scales which is shown for this model in Ref. [10]. The strong qualitative changes of the statistical aspects encoded in the particle distribution function have to be confronted with the comparably moderate changes of the spectral function shown in Fig. 4 and 5 .

\section{Equal-time spectral function and moments}

In order to make a connection with methods employed before, we discuss some equal-time properties of the spectral function. As has been discussed earlier, the spectral function is the commutator of fields and obeys elementary equal-time properties (2.12). There is a further hierarchy of equal-time relations that follow from the exact evolution equation (2.10). The identity

$$
\left.\partial_{x^{0}}^{2} \rho\left(x^{0}, y^{0} ; \mathbf{p}\right)\right|_{x^{0}=y^{0}}=0
$$

can also be traced back directly to the operator equation of motion. The following three equal-time derivatives are less trivial and read

$$
\begin{aligned}
\left.\partial_{x^{0}}^{3} \rho\left(x^{0}, y^{0} ; \mathbf{p}\right)\right|_{x^{0}=y^{0}}= & -\left[\mathbf{p}^{2}+M^{2}(x)\right] \\
\left.\partial_{x^{0}}^{4} \rho\left(x^{0}, y^{0} ; \mathbf{p}\right)\right|_{x^{0}=y^{0}}= & -2 \partial_{x^{0}} M^{2}(x) \\
\left.\partial_{x^{0}}^{5} \rho\left(x^{0}, y^{0} ; \mathbf{p}\right)\right|_{x^{0}=y^{0}}= & {\left[\mathbf{p}^{2}+M^{2}(x)\right]^{2}-3 \partial_{x^{0}}^{2} M^{2}(x) } \\
& -\left.\partial_{x^{0}} \Sigma_{\rho}\left(x^{0}, y^{0} ; \mathbf{p}\right)\right|_{x^{0}=y^{0}}
\end{aligned}
$$

These expressions are exact. One observes that the self energy $\Sigma_{\rho}$, necessary to describe scattering, enters for the first time only in the fifth derivative. This suggests that in formalisms which are strictly based on equal-time correlation functions [20], the kinetic aspects of scattering encoded in the spectral function may only be captured in very sophisticated truncations.

The above relations can be immediately recast in terms of moments of the spectral function in the Wigner presentation. We define the $n$ 'th moment as

$$
A_{n}\left(X^{0} ; \mathbf{p}\right)=\int_{-\infty}^{\infty} \frac{d \omega}{2 \pi} \omega^{n} \rho\left(X^{0} ; \omega, \mathbf{p}\right) .
$$


Due to the antisymmetry of $\rho\left(X^{0} ; \omega, \mathbf{p}\right)$ all even moments $A_{2 n}\left(X^{0} ; \mathbf{p}\right)$ vanish. It is straightforward to relate the odd moments directly to the equal-time derivatives given above, using $\partial_{x^{0}}=\frac{1}{2} \partial_{X^{0}}+\partial_{t}$. The first moment is just the sum rule, $A_{1}\left(X^{0} ; \mathbf{p}\right)=1$ and Eq. (6.1) provides a consistency check, $\left.\partial_{x^{0}}^{2} \rho\left(x^{0}, y^{0} ; \mathbf{p}\right)\right|_{x^{0}=y^{0}}=\partial_{X^{0}} A_{1}\left(X^{0} ; \mathbf{p}\right)=0$. For the higher derivatives one finds

$$
\begin{aligned}
& \left.\partial_{x^{0}}^{3} \rho\left(x^{0}, y^{0} ; \mathbf{p}\right)\right|_{x^{0}=y^{0}}=-A_{3}\left(X^{0} ; \mathbf{p}\right) \\
& \left.\partial_{x^{0}}^{4} \rho\left(x^{0}, y^{0} ; \mathbf{p}\right)\right|_{x^{0}=y^{0}}=-2 \partial_{X^{0}} A_{3}\left(X^{0} ; \mathbf{p}\right) \\
& \left.\partial_{x^{0}}^{5} \rho\left(x^{0}, y^{0} ; \mathbf{p}\right)\right|_{x^{0}=y^{0}}=-\frac{5}{2} \partial_{X^{0}}^{2} A_{3}\left(X^{0} ; \mathbf{p}\right)+A_{5}\left(X^{0} ; \mathbf{p}\right) .
\end{aligned}
$$

The combination of $(6.4)$ and (6.2) gives information on the time dependence of the lowest moments. Therefore, also in approximation schemes that are based on an expansion in moments, the lowest quantity that shows a direct dependence on the self energy $\Sigma_{\rho}$ appears only at relatively high (fifth) order. We conclude that both in equal-time formalisms and in approximations based on moments a proper inclusion of scattering effects might be difficult to achieve.

\section{Conclusion}

The presence of a nonequilibrium environment affects the quasiparticle structure of the theory, encoded in the spectral function. As a relevant physical application one may think of high energy heavy-ion collisions where spectral properties of the vector mesons are affected by the presence of nuclear matter.

In this paper we have concentrated on a self-consistent determination of the spectral function out of equilibrium, using a scalar field theory in $1+1$ dimensions as our (toy) model. We have calculated the nonequilibrium time evolution of the spectral function by solving the equations of motion obtained from the three-loop $2 P I$ effective action in real time. To compare the dynamics of the spectral function and the statistical aspects of the theory we also compute the evolution of an effective particle number distribution. Scattering and off-shell contributions are included in the dynamical evolution, in contrast to well-studied Hartree and leading-order large $N$ approximations. Even for moderate couplings and initial conditions far away 
from equilibrium, we observe a rather weak dependence of the Wigner transformed spectral function on $X^{0}$. This fact provides a necessary condition for a successful gradient expansion typically employed in the derivation of kinetic equations. After variations at early times we observe that the spectral function approaches a rather stable shape characterized by a nonvanishing width. A consistent "quantum Boltzmann equation" certainly has to relax the zero-width assumption employed in the derivation of the standard Boltzmann equation. We have argued that it might be notoriously difficult to derive the kinetic aspects of scattering encoded in the spectral function from equal-time formalisms or approximation schemes based on an expansion in moments. We emphasize that the employed framework based on the $2 P I$ effective action allows for a consistent description of both the early and the late time behavior. Since a change in the position of the peak and width of the spectral function can be described consistently in this framework, it would be interesting to extend the study presented here to more realistic models for heavy-ion collisions.

Finally the analysis performed in this paper was largely motivated by the physics of relativistic heavy-ion collisions. We would like to stress, however, that the approach using the $2 P I$ effective action is general and that possible applications of the formalism range from the early universe to condensed matter systems.

\section{Acknowledgements}

J.B. thanks Jürgen Cox for collaboration on related subjects. We would like to thank Tomislav Prokopec for many discussions. This work was supported by the TMR network Finite Temperature Phase Transitions in Particle Physics, EU contract no. FMRX-CT97-0122.

\section{References}

[1] J. Stachel, Nucl. Phys. A 654 (1999) 119C nucl-ex/9903007; R. Rapp and J. Wambach, nucl-th/0001014.

[2] For a recent review see e.g. W. Buchmüller and M. Plümacher, hepph/0007176.

[3] H.T.C. Stoof, J. Low Temp. Phys. 114 (1999) 11 cond-mat/9805393. 
[4] L.P. Kadanoff and G. Baym, "Quantum Statistical Mechanics", Benjamin, New York (1962).

[5] E. Calzetta and B. L. Hu, Phys. Rev. D 37 (1988) 2878.

[6] S. Mrowczynski and P. Danielewicz, Nucl. Phys. B342 (1990) 345;

S. Mrowczynski and U. Heinz, Annals Phys. 229 (1994) 1.

[7] J. Blaizot and E. Iancu, hep-ph/0101103.

[8] Y. B. Ivanov, J. Knoll and D. N. Voskresensky, Nucl. Phys. A672 (2000) 313 nucl-th/9905028; S. Leupold, Nucl. Phys. A672 (2000) 475 nuclth/9909080].

[9] M. Joyce, K. Kainulainen and T. Prokopec, Phys. Lett. B474 (2000) 402 hep-ph/9910535.

[10] J. Berges and J. Cox, hep-ph/0006160.

[11] G. Aarts and J. Berges, in preparation.

[12] R. D. Pisarski, hep-ph/9710370; D. Boyanovsky, H. J. de Vega, R. Holman, S. Prem Kumar and R. D. Pisarski, Phys. Rev. D 57 (1998) 3653 hep-ph/9711258.

[13] J. Schwinger, J. Math. Phys. 2 (1961) 407; L. V. Keldysh, Zh. Eksp. Teor. Fiz. 47 (1964) 1515.

[14] J. M. Cornwall, R. Jackiw and E. Tomboulis, Phys. Rev. D 10 (1974) 2428.

[15] K. Chou, Z. Su, B. Hao and L. Yu, Phys. Rept. 118 (1985) 1.

[16] G. Aarts and J. Smit, Nucl. Phys. B511 (1998) 451 hep-ph/9707342.

[17] M. Le Bellac, "Thermal Field Theory", Cambridge University Press, Cambridge (1996).

[18] G. Aarts and J. Smit, Phys. Rev. D 61 (2000) 025002 hep-ph/9906538.

[19] M. Sallé, J. Smit and J. C. Vink, hep-ph/0012362, hep-ph/0012346. 
[20] C. Wetterich, Phys. Rev. Lett. 78 (1997) 3598 hep-th/9612206];

G. Aarts, G. F. Bonini and C. Wetterich, Phys. Rev. D 63 (2001) 025012 [hep-ph/0007357. 